\title{
Adjunctive intrauterine Foley's Catheter Balloon adding to hypogastric artery ligation in the conservative management of morbid adherent placenta previa
}

\author{
Hany F. Sallam*, Nahla W. Shady \\ Department of Obstetrics and Gynecology, Aswan College of Medicine, Aswan, Egypt
}

Received: 23 March 2018

Accepted: 01 May 2018

*Correspondence:

Dr. Hany F. Sallam,

E-mail: hany.farouk@aswu.edu.eg

Copyright: ( ) the author(s), publisher and licensee Medip Academy. This is an open-access article distributed under the terms of the Creative Commons Attribution Non-Commercial License, which permits unrestricted non-commercial use, distribution, and reproduction in any medium, provided the original work is properly cited.

\begin{abstract}
Background: Objective of present study was to demonstrate the efficacy of Foley's catheters balloon tamponade as an adjuvant to control blood loss during and after a caesarian section in patient undergone hypogastric artery ligation (HAL) due to morbid adherent placenta previa (MAPP).

Methods: A single-center retrospective case-control clinical trial was carried out in a tertiary university hospital between March 2015 and March 2018, 124 women were undergoing cesarean delivery for MAPP, were managed conservatively either with HAL or HAL plus inflated Intrauterine Foley's Catheter Balloon to control post-partum hemorrhage secondary to MAPP. In the case of conservative treatment protocol failure, cesarean hysterectomy was performed.

Results: 124 women were enrolled ( $n=62$ in each group). group of women received HAL plus Intrauterine Foley's Catheter Balloon (group II)) showed a significant reduction in intraoperative and 4 hours post-operative blood loss compared with (Group I) which received HAL without Intrauterine Foley's Catheter Balloon. $(\mathrm{P}=0.0001,0.0 .015)$, so the overall estimated blood loss in group II showed significant reduction compared with group I $(\mathrm{P}=0.0001)$.

Conclusions: Adjunctive intrauterine Foley's catheter balloon adding to HAL ligation in the management of MAPP is a novel combination approach have proved to be effective to control of intraoperative and PPH and to preserve the uterus as possible and change in the concept of MAPP management always mandates hysterectomy. And may become an alternative valid option to planned cesarean-hysterectomy in well-selected cases who have a strong desire for future fertility.
\end{abstract}

Keywords: Foley's Catheter Balloon, Hypogastric artery ligation, Morbid adherent placenta previa

\section{INTRODUCTION}

MAPP represents one of the most morbid conditions in obstetrics, with high rates of hemorrhage, hysterectomy, and intensive care unit admission. ${ }^{1}$

The incidence of MAPP has progressively risen worldwide from one in 2500 pregnancies to approximately one in 500, mainly due to the increasing rates of cesarean section. Placenta previa in patients with two or more previous cesarean sections raises the risk for MAPP to $40-60 \%{ }^{2}$

While there is a debate about the optimal surgical management of women with MAPP, regardless of the strategy chosen, treatment is associated with substantial morbidity and mortality. Cesarean-hysterectomy is considered the gold standard treatment for the MAPP but 
is associated with high rates of severe maternal morbidity (40\%-50\%), with reported mortality rates up to $7 \% .^{3-6}$ Moreover, might not be considered first-line treatment for women who have a strong desire for future fertility.

The main types of conservative management which have been described: extirpative treatment, expectant management or leaving the placenta in situ, one-step conservative surgery, and the Triple-P procedure. ${ }^{7-10}$

For several decades, hypogastric artery ligation (HAL) has been used as an effective means of controlling intractable intraoperative or postpartum hemorrhage and contributing to the improvement of maternal outcome. The aim was to reduce the blood supply to the uterus and to prevent or help to arrest postpartum hemorrhage. ${ }^{11}$ Also, Balloon tamponade catheters have been used in the setting of a cesarean delivery for placenta previa. ${ }^{12}$

Till now there are controversies about the best conservative management strategies of MAPP, although primary objective in MAPP is to prevent bleeding, the ideal treatment of MAPP still elusive and debatable. With the rising incidence of abnormal placentation, it is important to define management strategies with a focus on reducing maternal morbidity and mortality mainly decrease PPH. ${ }^{13}$

So, the aims of our study to assess the additive role of Intrauterine inflated Foley's Catheter Balloon tamponade the lower uterine segment (the placental bed) on measured blood loss during and after a caesarian section in patient undergone hypogastric artery ligation due to MAPP.

\section{METHODS}

The study was conducted in accordance with the Declaration of Helsinki and was approved by the local departmental Ethics Committee. Informed written consent was obtained from all patients prior to their enrollment in this study.

This was a single center retrospective case-control study conducted during the period from March 2015 through March 2018 in Aswan University Hospital in Aswan, Egypt. which is a tertiary referral medical center for highrisk pregnancy. The study was approved by Institutional research board.

Study inclusion criteria were women undergoing elective cesarean delivery for MAPP. Diagnosis of MAPP based on ultrasound in which one or more of ultra-sonographic features were present: 1 - loss of the retroplacental clear space. 2- thinning of the myometrium overlying the placenta- 3-multiple irregular placental lacunae with a "moth-eaten" or "Swiss cheese" appearance of placenta.4- turbulent blood flow through the lacunae on Doppler velocimetry. This ultra-sonographic feature confirmed at cesarean section by placental adherence requiring digital separation.

Women with any of the following criteria were excluded from the study: 1-Patients with a cardiac, hepatic, renal or thromboembolic disease. 2-patients with placenta percreta (ultrasound features showed invasion of the placenta into the bladder or increased vascularity of the uterine serosa-bladder interface). 3-patient show to have placenta previa without signs of invasion (placenta previa without accreta) or those with a low-lying placenta and marginal placenta previa.4- women with inappropriate data.

All women were informed about the study of the beneficial and possible adverse effects of the conservative management using HAL, the technique of Intrauterine inflated Foley's Catheter Balloon tamponade the lower uterine segment and the risk of intraoperative hemorrhage and $\mathrm{PPH}$, the need for blood products transfusion, and the possibility of cesarean hysterectomy if needed to control severe bleeding. A written informed consent was taken from each woman before performing any intervention. Moreover, a written permission was obtained from each woman to use her data for research purposes.

\section{Intervention}

Eligible participants were allocated to one of two groups. group 1 [62 patients received 20 IU oxytocin (syntocinon. Novartis company) IV infusion plus bilateral HAL after fetus delivery], group 2 [62 patients received $20 \mathrm{IU}$ oxytocin (syntocinon. Novartis company) IV infusion plus bilateral internal iliac artery ligation after fetus delivery plus insertion of inflated follies catheter balloon]

Elective CS was planned at 37-38 weeks of gestation. An adequate amount of blood products was prepared to be available for transfusion. Delivery was performed by the same multidisciplinary team, including two expert obstetricians, an assistant, an expert anesthesiologist, and a pediatrician. All participants received general anesthesia. Two 16-gauge intravenous (IV) cannulas were inserted in 2 large forearm veins before induction of anesthesia, and one 16-gauge IV cannula was inserted in the external jugular vein after induction of anesthesia.

The abdomen was exposed through Pfannenstiel incision, after skin incision, the subcutaneous fat and abdominal fascia were opened crosswise, and the rectus muscle was opened on the midline, the parietal peritoneum was opened longitudinally, the visceral peritoneum was opened transversely and dissected downwards with the bladder and kept against symphysis pubis by a Doyen retractor, followed by transverse incision of the uterus at the upper border of the placenta to avoid transplacental incision which provoke severe bleeding. The fetus was delivered. Bilateral HAL was done before placental delivery by Transabdominal/Trans peritoneal Approach. 
Some right-angle forceps were used to separate the plane between artery and vein and the artery was ligated with suture ties on both sides. 20 IU oxytocin was given IV infusion after HAL to prevent premature separation of placenta which provoked severe bleeding then placenta delivery was done.

In the Foley's group After acceptable control of bleeding from the placental bed, the internal os of the cervix was identified and a double-way 20 Fr Foley's catheter with a 30-50-ml balloon (Amecath, Ameco Medical Industries, 10th of Ramadan City, Egypt) was inserted through the cervix to be handled by an assistant through the vagina and fixed to the patient's lower limb after inflation of the catheter balloon by $80 \mathrm{ml}$ warm saline and pulling it against the lower uterine segment. Only one catheter was used for tamponade. The uterine incision was then repaired over the inflated balloon. A wide pore drain was then inserted in the Douglas pouch, and the abdominal wall was repaired. The intrauterine inflated Foley's catheter balloon was left in place for $24 \mathrm{~h}$ after the operation and then removed.

Finally, the uterus was closed in three layers and a pelvic drain was inserted in Douglas pouch in all patients followed by standard repair of the abdominal wall.

\section{Blood loss estimation}

Intraoperative blood loss was measured by adding the volume of the contents of the suction bottle and the difference in weight (in grams) between the dry and the soaked operation sheets and towels $(1$ gram $=1 \mathrm{ml}$.). Post-operative blood loss was measured through the volume of the contents of the pelvic drain which measured every 12 hours and on removing the drain and the difference in weight (in grams) between the dry and the soaked vaginal pads after 4 hours post-operative (1 gram $=1 \mathrm{ml})$. After that the total blood loss was calculated by the addition of intraoperative and postoperative blood loss.

\section{Study outcome}

The primary outcome was an estimation of intraoperative, postoperative and total blood loss $(\mathrm{ml})$. The secondary outcome measures included pulse rate, blood pressure and temperature which recorded continuously intraoperative, then every 30 minutes after operation therefor the mean were calculated. Also measures included the need for blood transfusion $\geq 4$ units, operative time, period for hospitalization, cesarean hysterectomy, bladder injury, 24 hours postoperative hemoglobin concentration. After collecting all the data, the data were tabulated and analyzed.

\section{Statistically analysis}

Data were entered and statistically analyzed using the Statistical Package for Social Sciences (SPSS) version
16. Qualitative data were described as numbers and percentages. Chi-square test, Fisher's Exact test, and delete Monte Carlo test were used for comparison between groups, as appropriate.

Quantitative data were described as means (SD) or medians, as appropriate. They were tested for normality by Kolmogorov-Smirnov test.

In the normally distributed variables, independent sample t-test instead of one-way ANOVA test with LSD post-hoc multiple comparisons were used for comparison between groups, as appropriate.

In the non-normally distributed variables, Mann Whitney test was used for comparison between groups, as appropriate. Odds ratios and their 95\% confidence interval were calculated. $p$ value $\leq 0.05$ was statistically significant.

\section{RESULTS}

There was no significant difference between the two groups with respect to their age, weight, height, parity, gestational age, pre-operative pulse, systolic blood pressure (SBP), diastolic blood pressure (DBP), temperature, initial hemoglobin and number of previous cesarean sections (CS) (Table 1).

Table 1: Preoperative characteristics of pregnant women in the study groups.

\begin{tabular}{|c|c|c|c|}
\hline Parameters & $\begin{array}{l}\text { Group I } \\
(n=62)\end{array}$ & $\begin{array}{l}\text { Group II } \\
(\mathrm{n}=62)\end{array}$ & Significance \\
\hline Age (year) & $29.71 \pm 2.84$ & $29.68 \pm 2.53$ & 0.774 \\
\hline Weight (kg) & $78.68 \pm 5.48$ & $78.13 \pm 4.92$ & 0.891 \\
\hline Parity & $3(2-5)$ & $3(2-5)$ & 0.707 \\
\hline Hight & $163.03 \pm 3.89$ & $164.52 \pm 4.55$ & 0.375 \\
\hline Pulse & $82.19 \pm 5.03$ & $81.65 \pm 5.3$ & 0.795 \\
\hline Temperature & $37.0 \pm 0.14$ & $36.97 \pm 0.18$ & 0.709 \\
\hline SBP & $122.1 \pm 2.52$ & $122.13 \pm 2.7$ & 0.889 \\
\hline DBP & $80.74 \pm 3.14$ & $80.26 \pm 3.23$ & 0.685 \\
\hline $\begin{array}{l}\text { Initial } \\
\text { Hemoglobin } \\
(\%)\end{array}$ & $9.88 \pm 0.67$ & $9.91 \pm 0.7$ & 0.874 \\
\hline $\begin{array}{l}\text { Gestational } \\
\text { age (weeks) }\end{array}$ & $35.58 \pm 0.62$ & $35.55 \pm 0.77$ & 0.977 \\
\hline $\begin{array}{l}\text { Previous } \\
\text { cesarean } \\
\text { section }(\mathrm{CS})\end{array}$ & $2.97 \pm 0.84$ & $2.87 \pm 0.85$ & 0.906 \\
\hline
\end{tabular}

SBP (Systolic Blood Pressure), DBP (Diastolic Blood Pressure), CS (Cesarean Section); \# Variables are presented as mean and standard deviation, median (minimum - maximum). Statistical significance if $\mathrm{p}$ value $\leq 0.05$

There was a great reduction in intraoperative blood loss in group II $(813.23 \pm 194.07)$ compared with Group I (1283.55 \pm 315.36$),(\mathrm{P}=0.0001)$.

Also, there was a great reduction in postoperative bleeding either vaginal bleeding or blood in an 
intraabdominal drain in group II than group I $(\mathrm{p}=0.015$ and 0.0001 respectively) Hence the total estimated blood loss in group II $(1151.61 \pm 246.38)$ showed highly reduction compared with group I $(1800 \pm 980)(\mathrm{P}=0.0001)$ (Table 2).

Table 2: Primary outcome in the study groups.

\begin{tabular}{|llll|}
\hline Variables & Group I $(\mathrm{n}=62)$ & Group II $(\mathrm{n}=62)$ & Significance \\
\hline Blood loss & & & $0.0001^{*}$ \\
\hline Intraoperative & $1283.55 \pm 315.36$ & $813.23 \pm 194.07$ & $0.015^{*}$ \\
\hline Postoperative vaginal bleeding & $260(110-330)$ & $200(130-160)$ & $0.0001^{*}$ \\
\hline Blood in drains & $160(110-330)$ & $130(100-350)$ & $0.0001^{*}$ \\
\hline Total blood loss & $1800 \pm 980$ & $1151.61 \pm 246.38$ & 0.269 \\
\hline No of cesarean hysterectomy $(\%)$ & $10(16.1)$ & $3(4.8)$ & \\
\hline
\end{tabular}

*Statistical significant difference; \# Variables are presented as mean and standard deviation, median(minimum-maximum) and number (percentage).

The number of patients needs blood transfusion $\geq 4$ bags were a significant increase in group I; [34 patients $(54.8 \%)$ ] versus 8 patients in group II (12.9\%).
$(\mathrm{P}=0.0001)$. Also, the number of patients need admission to ICU were a significant increase in group I [18 patients $(29 \%)]$ versus 2 patients in group II $(3.2 \%)(\mathrm{P}=0.006)$.

Table 3: Secondary outcome in the study groups.

\begin{tabular}{|llll|}
\hline Variables & Group I $(\mathrm{n}=62)$ & Group II $(\mathrm{n}=62)$ & Significance \\
\hline Pulse & $93.52 \pm 10.05$ & $88.0 \pm 6.38$ & $0.006^{*}$ \\
\hline Temperature & $36.95 \pm 0.16$ & $36.95 \pm 0.16$ & 0.918 \\
\hline SBP & $114.23 \pm 7.71$ & $120.52 \pm 2.54$ & $0.0001^{*}$ \\
\hline DBP & $73.87 \pm 5.58$ & $77.26 \pm 3.38$ & $0.002^{*}$ \\
\hline Post op Hemoglobin $(\%)$ & $9.16 \pm 0.56$ & $9.37 \pm 0.61$ & 0.159 \\
\hline NO of ICU admission & $18(29.0)$ & $2(3.2)$ & $0.006^{*}$ \\
\hline Bladder injury & $6(9.7)$ & $4(6.5)$ & 0.897 \\
\hline Need Blood Transfusion $>4$ unit & $34(54.8)$ & $8(12.9)$ & $0.0001^{*}$ \\
\hline Nausea & $6(9.7)$ & $12(19.4)$ & 0.652 \\
\hline Vomiting & $2(3.2)$ & $4(6.5)$ & 0,875 \\
\hline Diarrhea & $2(3.2)$ & $4(6.5)$ & 1.00 \\
\hline Operative times & $101.94 \pm 11.6$ & $98.16 \pm 9.83$ & 0.330 \\
\hline Hospital stays & $4.9 \pm 1.5$ & $3.87 \pm 0.85$ & $0.001^{*}$ \\
\hline
\end{tabular}

SBP (Systolic Blood Pressure), DBP (Diastolic Blood Pressure); *Statistical significant difference; \#Variables are presented either mean and standard deviation and number (percentage).

There was significant increase in postoperative hospital stay in group I $(4.9 \pm 1.5)$ compared with group II $(3.87 \pm 0.85)(\mathrm{p}=0.001)$. There was a significant increase in post-operative pulse in group I $(93.52 \pm 10.05)$ compared with Group II $(88.0 \pm 6.38)$. $(\mathrm{P}=0.006)$. Also, there was a significant decrease in both post-operative SBP (114.23 \pm 7.71$)$ and DBP $(73.87 \pm 5.58)$ in group I compared with SBP $(120.52 \pm 2.54)$ and DBP $(77.26 \pm 3.38)$ in group II $(\mathrm{P}=0.0001$ and 0.002 respectively).

There was no significant difference between both groups with respect to post-operative temperature, postoperative hemoglobin, the incidence of cesarean hysterectomy, the incidence of urinary bladder injury, operation time, the incidence of nausea, vomiting, and diarrhea $(\mathrm{P}=0.918$, $0.159,0.269,0.897,0.330,0.652,0.875$ and 1.00 respectively) (Table 2 and 3 ).

\section{DISCUSSION}

In the current study, prophylactic use of HAL plus Intrauterine Foley's Catheter Balloon is effectively reduced intra-operative, post-operative blood loss, blood transfusion needs as well as lower the incidence of PPH due to MAPP as compared with hypogastric artery ligation alone. Also, associated with a decrease in cesarean hysterectomy for post-partum hemorrhage. 


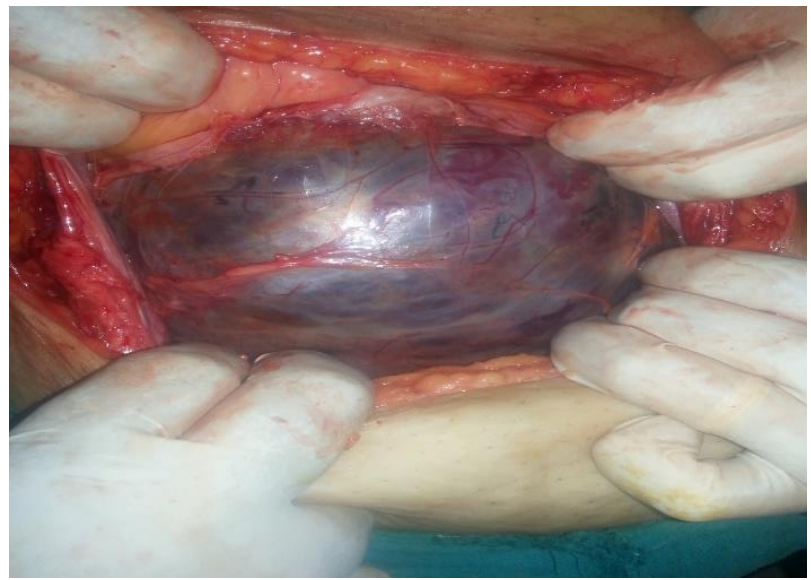

Figure 1: Morbid adherent placenta previa.

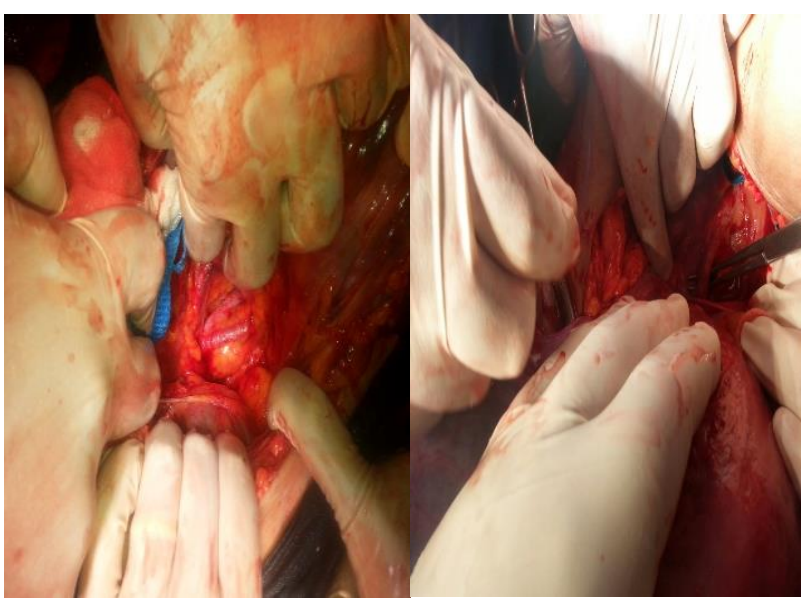

Figure 2: Demonstration of HA.

In the best of our knowledge, we claim that present study was the first to evaluate the novel combination of Intrauterine Foley's Catheter Balloon during cesarean section for MAP adjunctive to HAL for the aim of prevention of intraoperative and post-partum hemorrhage with aiming for conservative management. This strategy aims to combine the advantages of the conservative approach (i.e., preserving fertility) and of cesareanhysterectomy (no persistent high risk of bleeding or infection after the procedure).

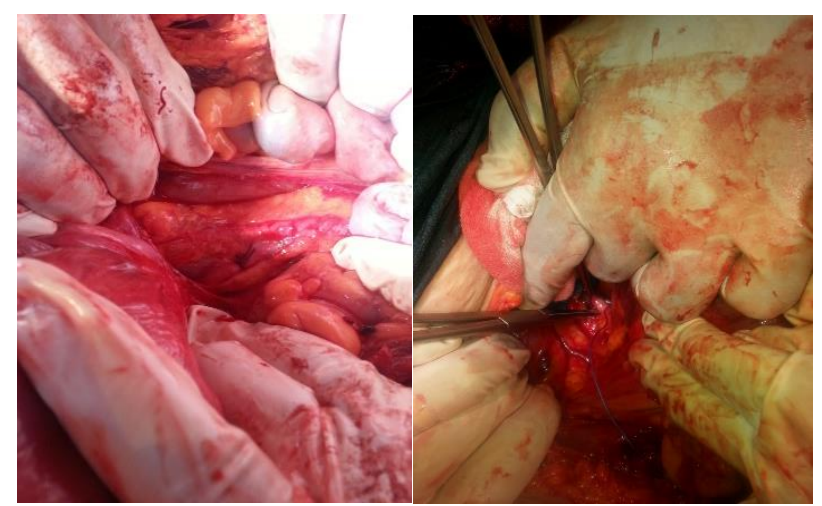

Figure 3: Ligation of HA.

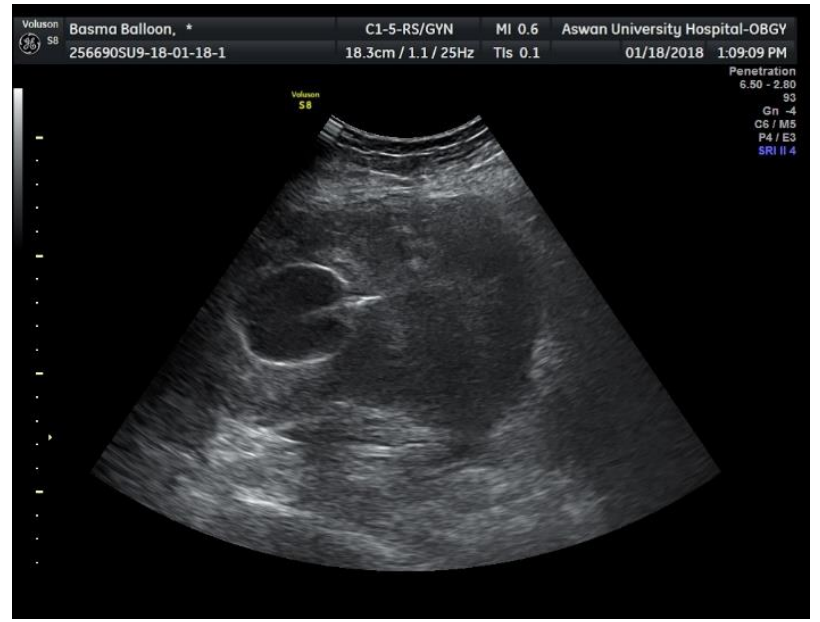

Figure 4: TVS show Foley's catheter balloon tamponade at the lower uterine segment.

Hypogastric artery ligation will control blood loss by reducing arterial pulse pressure and decreasing distal blood flow by converting the pelvic arterial circulation into a venous system. ${ }^{13}$ The rationale for this is based on the hemodynamic studies of Burchell, which showed that hypogastric artery ligation reduced pelvic blood flow by half and pulse pressure by $85 \%$, resulting in venous pressures in the arterial circuit thus promoting hemostasis. ${ }^{11}$ However, the reported success rate of hypogastric artery ligation varies from 40 to $100 \%$, and the procedure prevents hysterectomy in only $50 \%$ of cases. $^{14}$

Bailit et al. showed that women with prenatally suspected MAPP 33\% had massive blood loss and 92\% need for hysterectomy despite uterine artery ligation, hypogastric artery ligation, B-Lynch suture, balloon tamponade. ${ }^{15}$

Chandraharan et al. described their procedure as triple $\mathrm{P}$, and they reported remarkably low blood loss, ranging from 800 to $1500 \mathrm{~mL}$ per patient. ${ }^{16}$

Fitzpatrick et al highlighted that avoiding any attempt to remove the placenta, with the aim of uterine preservation reduces the severity of hemorrhage. ${ }^{14,20}$

Eller AG suggested a stepwise approach, including transverse incision in the uterus at the upper border of the placenta, delayed extraction of the placenta after transient bilateral kinking of the uterine arteries and bilateral ligation of the anterior division of the IIA, proper identification of the lower uterine segment (LUS), and repair of the uterine incision. ${ }^{9}$

Eller AG highlight attempts at placental removal before hysterectomy, and hypogastric artery ligation significantly increased early maternal morbidity (67 versus $36 \%, \mathrm{P}=0.04) .{ }^{9}$ Importantly, fertility preservation was not achieved in any case of attempted placental removal among women with antenatally suspected 
MAPP. ${ }^{9}$ In present study hypogastric artery ligation before attempt to remove the placenta preserve fertility in $84 \%$ of the group I and adding Foley's Catheter Balloon further preserve fertility in $97 \%$ of the group II.

Cesarean hysterectomy is one of the recommended management options for MAPP, however, this approach might not be considered first-line treatment for women who have a strong desire for future fertility. ${ }^{14}$ The main aim of conservative management of PA is to reduce operative injury and blood loss and to increase the chance that a hysterectomy can be avoided in present study, authors tried to preserve the uterus by ligation of hypogastric artery alone or plus compressing the placental bed by Foley's catheter balloon filled with 80 ml warm saline, which was held in place against the lower uterine segment by an assistant then by its fixation to the thigh of the patient just after removal of the placenta. All other procedures to control bleeding were done as usual when needed. In present study, group allocated to hypogastric artery ligation alone only 10 cases from 62 need hysterectomy $(16.1 \%)$ and group allocated to hypogastric artery ligation plus balloon catheter only2 cases from 62 need hysterectomy (3.2\%).

In cases of placenta previa, bleeding vessels present in the lower uterine segment could not be compressed by uterine contraction; so, it is logic that decrease blood supply to the uterus by ligation of hypogastric artery alone or in combination of rapid compression of the lower uterine segment with the inflated balloon just after removal of the placenta could decrease the blood loss during the operation and give our team the time needed to do the other conservative measures without deterioration of the patient's general condition. Similar results were obtained when Maher and Abdelaziz used Bakri balloon in cases of placenta previa after the occurrence of PPH. ${ }^{18}$

Uterine compression after delivery can be done by many tools and different types of catheters. The Bakri balloon could not be used except after closure of the uterine incision to compress the entire uterine cavity. ${ }^{18}$ The Foley's catheter could be used intraoperatively before the occurrence of severe blood loss. It has a small capacity, but when it was applied to the lower uterine segment only, it was effective in controlling bleeding in cases of MAP. Its effect may be attributable to a hydrostatic pressure effect on the lower uterine segment. The catheter also allows drainage of blood to enable to calculate postoperative blood loss. There is growing evidence supporting the use of inflated Foleys catheter balloon tamponade in the management of postpartum hemorrhage, often when other measures have failed and desire of preserve the uterus is warranted. ${ }^{19}$

Rauf et al who preserved the uterus in $84.3 \%$ of cases with MAPP by performing IIA ligation and endo-uterine hemostatic sutures. ${ }^{13}$
The strengths of present study include that being the first randomized study that described the use of intrauterine inflated Foley's catheter balloon plus hypogastric artery ligation for control of PPH during CS in cases of PPA. Foley's Catheter Balloon is considered a low-cost alternative to the other types of intrauterine catheters and may be suitable for use in low-resources developing countries.

The study had its limitations. First, the small sample size of included women in the study groups may limit the generalizability of our results.

The second, limitation of present study was probably related to the lack of blinding and randomization. Finally, limitations of present study include those inherent to retrospective nature. Future studies designed prospectively with a large sample size need to be performed to further clarify the place of $\mathrm{N}$ and $\mathrm{H}$ technique in the conservative management of MAPP.

\section{CONCLUSION}

Authors conclude that, Inflated Intrauterine Foley's Catheter Balloon Adding to internal iliac artery ligation in the Management of MAPP is a novel approach have proved to be efficient to control of intraoperative and PPH and preserve the uterus as possible and change in the concept of Placenta accrete management always mandates hysterectomy. And may become an alternative valid option to planned cesarean-hysterectomy in wellselected cases who have a strong desire for future fertility.

\section{Funding: No funding sources Conflict of interest: None declared}

Ethical approval: The study was approved by the Institutional Ethics Committee

\section{REFERENCES}

1. Grobman WA, Bailit JL, Rice MM et al. Frequency of and factors associated with severe maternal morbidity. Obstet Gynecol. 2014 Apr;123(4):804-10.

2. Pinas-Carrillo A, Chandraharan E. Management of morbidly adherent placenta. Obstet Gynaecol Reproduct Med. 2016 Oct 1;26(10):283-90.

3. Sentilhes L, Vayssière C, Deneux-Tharaux C, Aya AG, Bayoumeu F, Bonnet MP, Djoudi Ret al. Postpartum hemorrhage: Guidelines for clinical practice from the French College of Gynaecologists and Obstetricians (CNGOF) in collaboration with the French Society of Anesthesiology and Intensive Care (SFAR). Eur J Obstet Gynecol Biol Reprod. 2016;198:12-21.

4. Sentilhes L, Goffinet F, Kayem G. Management of placenta accreta. Acta Obstet Gynecol Scand. 2013;92:1125-34.

5. Alchalabi HA, Lataifeh I, Obeidat B, Zayed F, Khader YS, Obeidat N. Morbidly adherent placenta 
previa in current practice: prediction and maternal morbidity in a series of 23 women who underwent hysterectomy. J Matern Fetal Neonatal Med. 2014;27(17):1734-7.

6. Royal College of Obstetricians and Gynaecologists (RCOG). Placenta praevia, placenta praevia accreta and vasa praevia: diagnosis and management (Greentop Guideline No. 27). London: RCOG;2011:26.

7. Kayem G, Davy C, Goffinet F, Thomas C, Clement D, Cabrol D. Conservative versus extirpative Management in cases of placenta accreta. Obstet Gynecol. 2004;104:531-6.

8. Sentilhes L, Ambroselli C, Kayem G, et al. Maternal outcome after conservative treatment of placenta accreta. Obstet Gynecol. 2010;115:526-34.

9. Eller AG, Porter TF, Soisson P, Silver RM. Optimal management strategies for placenta accreta. BJOG. 2009;116:648-54.

10. Teixidor Vinas M, Belli AM, Arulkumaran S, Chandraharan E. Prevention of postpartum hemorrhage and hysterectomy in patients with morbidly adherent placenta: A cohort study comparing outcomesbbefore and after introduction of the Triple-P procedure. Ultrasound Obstet Gynecol. 2015; 46:350-5.

11. Burchell RC. Physiology of internal iliac artery ligation. J Obstet Gynaec Brit Cwlth. 1968;75:64251 .

12. Bakri YN. Uterine tamponade-drain for hemorrhage secondary to placenta previa-accreta. Int $\mathbf{J}$ Gynecol Obstet. 1992;37(4):302-3.

13. Rauf M, Ebru C, Sevil E, Selim B. Conservative management of post-partum hemorrhage secondary to placenta previa-accreta with hypogastric artery ligation and endo-uterine hemostatic suture. J Obstet Gynaecol Res. 2017 Feb 1;43(2):265-71.
14. Shakur H, Roberts I, Bautista R, Collaborators C-T. Effects of tranexamic acid on death, vascular occlusive events, and blood transfusion in trauma patients with significant hemorrhage (CRASH-2): a randomized, placebo-controlled trial. Lancet 2010;376:23-32.

15. Bailit JL, Grobman W, Rice MM, Reddy UM, Wapner RJ, Varner MW et al. Morbidly adherent placenta treatments and outcomes. Obstet Gynecol. 2015;125:683-9.

16. Chandraharan E, Rao S, Belli AM, Arulkumaran S. The triple-P procedure as a conservative surgical alternative to peripartum hysterectomy for placenta percreta. Int J Gynaecol Obstet. 2012;117:191-4.

17. Fitzpatrick KE, Sellers S, Spark P, Kurinczuk JJ, Brocklehurst P, Knight M. The management and outcomes of placenta accreta, increta, and percreta in the UK: a population-based descriptive study. BJOG. 2014 Jan;121(1):62-71.

18. Maher MA, Abdelaziz A. Comparison between two management protocols for postpartum hemorrhage during cesarean section in placenta previa: balloon protocol versus non-balloon protocol. J Obstet Gynaecol Res. 2017;43(3):447-55.

19. Dabelea V, Schultze PM, McDuffie RS. Intrauterine balloon tamponade in the management of postpartum hemorrhage. Am J Perinatol. 2007;24(06):359-64.

Cite this article as: Sallam HF, Shady NW. Adjunctive intrauterine Foley's Catheter Balloon adding to hypogastric artery ligation in the conservative management of morbid adherent placenta previa. Int J Reprod Contracept Obstet Gynecol 2018;7:2093-9. 